\title{
On the Statistical Properties of Generalized Rice Multipath Fading Channels
}

\author{
Néji Yousșef*, Cheng-Xiang Wang ${ }^{\dagger}$, Matthias Pätzold ${ }^{\dagger}$, Insaf Jaafar* and Sami Tabbane* \\ * Ecole Supérieure des communications de Tunis \\ Cité El Ghazala, 2083 Ariana, Tunis, Tunisia \\ Email: neji.youssef@supcom.rnu.tn, sami.tabbane@supcom.rnu.tn \\ $\dagger$ Faculty of Engineering and Science, Agder University College \\ Grooseveien 36, N-4876, Grimstad, Norway \\ Email: cheng.wang@hia.no, matthias.paetzold@hia.no
}

\begin{abstract}
Statistical properties of generalized Rice multipath fading channels are studied. A closed-form expression for the probability density function (PDF) of the phase process is derived first. Second order statistics, in the form of the level-crossing rate (LCR) and the average duration of fades (ADF), are then investigated for an arbitrary crossing level of the fading amplitude. Additionally, it is shown that the generalized Rice model yields a good fitting to available measurement data on mobile satellite channels in the case of an environment with light shadowing.
\end{abstract}

\section{INTRODUCTION}

Rayleigh and Rice processes are the most widely used models in the statistical description of the behavior of smal scale fading in multipath propagation channels [1], [2], [3]. These processes are generated by using two uncorrelated Gaussian processes having identical variances. In order to increase the flexibility of these classical models, so that they enable a better statistical fitting to real-world mobile fading channels, modifications of the these models have been studied in [4], [2], where the Gaussian processes describing the models are allowed to be cross correlated. In [2], it was shown that such extended models allow a better description of real-world mobile fading channels.

Another possibility enabling the extension of the classical Rayleigh and Rice models, while keeping the basic concept used for their generation, is to assume that the underiying Gaussian processes can have different variances. Accordingly, the Hoyt process [5] can be regarded as an extension of the Rayleigh process derived from two Gaussian processes with different variances. The first order statistics of the Hoyt model is studied in [5], while the second order statistics is analyzed in [6]. Additionally, a comparison of the first and second order statistics of the Hoyt model with measurement data [6] has shown the ability of the model to describe the statistics of real-world mobile fading channels for the case of non-line-ofsight (NLOS) reception.

A statistical model was proposed in [7] that is suitable for environments where a LOS component is present. This model assumes that the multipath component is Hoyt distributed and the LOS component has a non-zero value constant phase shift. The so-called generalized Rice model (or Beckmann model
[7]) was also subsequently studied in [8], where the PDF of the envelope was re-derived and combined with a lognormal process to obtain a model that takes the effects of small scale fading and shadowing into account. Furthermore, in [8] the theoretical PDF of the resultant process was shown to fit accurately to measurement data on mobile satellite channels. In [9], the moment generating function of the generalized Rice process, which plays an important role in the performance analysis of transmission systems, is obtained in closed form. In this paper, we study the statistics of the generalized Rice fading channel model by deriving the PDF of the phase process, and the second order statistics of the envelope fading, i.e., the LCR and the ADF. The fitting of the first and second order statistics of the underlying fading model to available measurement data of mobile satellite channels is also considered for the case of an environment with light shadowing. In addition to the results reported in [8], it is demonstrated that the generalized Rice model is useful to describe accurately the statistics of realistic mobile radio channels.

The remainder of the paper is organized as follows. The analytical generalized Rice model is described in Section II. Section III contains the derivation of the PDF of the phase process, the LCR and the ADF. Comparison of the derived statistics to corresponding measurement data is reported in Section IV. Finally, Section V contains the conclusion of the paper.

\section{THE GENERALIZED RICE FADING MODEL}

The complex envelope of a received signal in a generalized Rice fading environment is described by the complex Gaussian process given by

$$
\mu(t)=A \exp \left(j \theta_{0}\right)+\mu_{1}(t)+j \mu_{2}(t)
$$

where $A$ is the amplitude and $\theta_{0}$ is a constant phase shift of the LOS component, while $\mu_{1}(t)$ and $\mu_{2}(t)$ are uncorrelated zero mean real-valued lowpass Gaussian processes having the variances $\sigma_{1}^{2}$ and $\sigma_{2}^{2}\left(\sigma_{1}^{2} \neq \sigma_{2}^{2}\right)$, respectively. The amplitude, $R(t)$, of the generalized Rice fading process is then obtained as the modulus of $\mu(t)$ according to 


$$
\begin{aligned}
R(t) & =\left|A \exp \left(j \theta_{0}\right)+\mu_{1}(t)+j \mu_{2}(t)\right| \\
& =\sqrt{\left(A \cos \theta_{0}+\mu_{1}(t)\right)^{2}+\left(A \sin \theta_{0}+\mu_{2}(t)\right)^{2}}
\end{aligned}
$$

It is worth mentioning that the assumption of having different mean values for the in-phase and quadrature components of the Gaussian process defined by (1) can be explained by the non-zero value of the phase shift $\theta_{0}$. Similarly as for the Rice channel, the generalized Rice factor is defined as [9]

$$
K=\frac{A^{2}}{\sigma_{1}^{2}+\sigma_{2}^{2}} .
$$

The phase variation, which is important to the study of coherent communication systems, is described by the phase process $\vartheta(t)=\arg (\mu(t))$ according to

$$
\vartheta(t)=\arctan \left(\frac{A \sin \theta_{0}+\mu_{2}(t)}{A \cos \theta_{0}+\mu_{1}(t)}\right) .
$$

The derivation of the PDF of $\vartheta(t)$ as well as the study of the second order statistics of $R(t)$ will be the topic of the next section.

\section{THE STATISTICS OF THE GENERALIZED RICE MODEL}

The derivation of the first and second order statistics of the generalized Rice model requires first the investigation of the joint PDF (JPDF), $p_{R \dot{R} \vartheta \dot{\vartheta}}(z, \dot{z}, \theta, \dot{\theta})$, of the processes $R(t)$ and $\vartheta(t)$ and their time derivatives $\dot{R}(t)$ and $\dot{\vartheta}(t)$, respectively. This JPDF can be obtained from the multivariate Gaussian distribution of the processes $\mu_{1}(t), \mu_{2}(t), \dot{\mu}_{1}(t)$ and $\dot{\mu}_{2}(t)$ at the same time $t$. Here $\dot{\mu}_{1}(t)$ and $\dot{\mu}_{2}(t)$ are the time derivatives of the processes $\mu_{1}(t)$ and $\mu_{2}(t)$, respectively. In the case of processes $\mu_{i}(t)(i=1,2)$ with symmetrical Doppler power spectral densities, the JPDF of the Gaussian processes $\mu_{1}(t)$, $\mu_{2}(t), \dot{\mu}_{1}(t)$ and $\dot{\mu}_{2}(t), p_{\mu_{1} \mu_{2} \dot{\mu}_{1} \dot{\mu}_{2}}\left(x_{1}, x_{2}, \dot{x}_{1}, \dot{x}_{2}\right)$, is given by [2]

$$
\begin{aligned}
& p_{\mu_{1} \mu_{2} \dot{\mu}_{1} \dot{\mu}_{2}}\left(x_{1}, x_{2}, \dot{x}_{1}, \dot{x}_{2}\right)= \\
& \frac{\exp \left(-\left(\frac{x_{1}^{2}}{2 \sigma_{1}^{2}}+\frac{x_{2}^{2}}{2 \sigma_{2}^{2}}+\frac{\dot{x}_{3}^{2}}{2 \beta_{1}}+\frac{\dot{x}_{2}^{2}}{2 \beta_{2}}\right)\right)}{4 \pi^{2} \sigma_{1} \sigma_{2} \sqrt{\beta_{1}} \sqrt{\bar{\beta}_{2}}}
\end{aligned}
$$

where $\beta_{i}$ is the variance of the process $\dot{\mu}_{i}(t)(i=1,2)$. Furthermore, the transformation of the Cartesian coordinates $\left(x_{1}, x_{2}\right)$ to polar coordinates $(z, \theta)$ allows us to write

$$
\begin{aligned}
& x_{1}=z \cos \theta-A \cos \theta_{0}, \quad \dot{x}_{1}=\dot{z} \cos \theta-\dot{\theta} z \sin \theta \\
& x_{2}=z \sin \theta-A \sin \theta_{0}, \quad \dot{x}_{1}=\dot{z} \sin \theta+\dot{\theta} z \cos \theta .
\end{aligned}
$$

Next, using (5) and performing the variable transformation described by (6), allows to express the JPDF $p_{R \dot{R} \vartheta \dot{v}}(z, \dot{z}, \theta, \dot{\theta})$

$$
\begin{aligned}
& p_{R \dot{R} \vartheta \dot{\theta}}(z, \dot{z}, \theta, \dot{\theta})=\frac{z^{2}}{(2 \pi)^{2} \sigma_{1} \sigma_{2} \sqrt{\beta_{1} \beta_{2}}} \\
& \cdot \exp \left[-\frac{z^{2} \cos ^{2} \theta+A^{2} \cos ^{2} \theta_{0}-2 A z \cos \theta_{0} \cos \theta}{2 \sigma_{1}^{2}}\right. \\
& -\frac{z^{2} \sin ^{2} \theta+A^{2} \sin ^{2} \theta_{0}-2 A z \sin \theta_{0} \sin \theta}{2 \sigma_{2}^{2}} \\
& -\frac{\dot{z}^{2} \cos ^{2} \theta+z^{2} \dot{\theta}^{2} \sin ^{2} \theta-2 z \dot{z} \dot{\theta} \cos \theta \sin \theta}{2 \beta_{1}} \\
& \left.-\frac{\dot{z}^{2} \sin ^{2} \theta+z^{2} \dot{\theta}^{2} \cos ^{2} \theta+2 z \dot{z} \dot{\theta} \cos \theta \sin \theta}{2 \beta_{2}}\right]
\end{aligned}
$$

where $z \geq 0,-\infty<\dot{z}<\infty,-\pi \leq \theta<\pi$, and $-\infty<\dot{\theta}<$ $\infty$. The first and second order statistical properties can now be derived from (7).

\section{A. First order statistics}

The PDF of $R(t), p_{R}(z)$, is obtained from (7) according to

$$
p_{R}(z)=\int_{0}^{2 \pi} \int_{-\infty}^{\infty} \int_{-\infty}^{\infty} p_{R \dot{R} \vartheta \dot{\vartheta}}(z, \dot{z}, \theta, \dot{\theta}) d \dot{\theta} d \dot{z} d \theta
$$
8]

$$
\begin{aligned}
& p_{R}(z)=\frac{z}{2 \pi \sigma_{1} \sigma_{2}} \exp \left[-A^{2} h\left(\theta_{0}\right)\right] \\
& \int_{0}^{2 \pi} \exp \left[-z^{2} h(\theta)+A z g\left(\theta_{0}, \theta\right)\right] d \theta
\end{aligned}
$$

where

$$
h(\theta)=\frac{\cos ^{2} \theta}{2 \sigma_{1}^{2}}+\frac{\sin ^{2} \theta}{2 \sigma_{2}^{2}}
$$

and

$$
g\left(\theta_{0}, \theta\right)=\frac{\cos \theta_{0} \cos \theta}{\sigma_{1}^{2}}+\frac{\sin \theta_{0} \sin \theta}{\sigma_{2}^{2}} .
$$

From (9), we note that the phase shift, $\theta_{0}$, of the LOS component has an influence on the PDF $p_{R}(z)$. The influence of $\theta_{0}$ on $p_{R}(z)$ can be studied from Fig. 1. We should mention that, on contrary to what has been reported in [8], the PDF in (9) has in all cases a single maximum value. Obviously, it can be verified that the above PDF includes the Rayleigh [10] ( $\left.A=0, \sigma_{1}=\sigma_{2}\right)$, Rice [2] $\left(\sigma_{1}=\sigma_{2}\right)$, Hoyt [5] $(A=0)$ and the one-sided Gaussian [5] $\left(A=0, \sigma_{2}=0\left(\sigma_{1} \neq 0\right)\right.$ or $\sigma_{1}=0$ $\left(\sigma_{2} \neq 0\right)$ ) PDFs as special cases. Therefore, the underlying model is a generalization of those fading processes.

Similarly, the PDF of the phase process $\vartheta(t), p_{\vartheta}(\theta)$, can be derived by solving the integrals over the joint PDF $p_{R \dot{R} \vartheta \dot{\vartheta}}(z, \dot{z}, \theta, \dot{\theta})$ according to

$$
p_{\vartheta}(\theta)=\int_{-\infty}^{\infty} \int_{-\infty}^{\infty} \int_{0}^{\infty} p_{R \dot{R} \vartheta \dot{\vartheta}}(z, \dot{z}, \theta, \dot{\theta}) d z d \dot{z} d \dot{\theta}
$$


Performing the integrations in (12) using [11], yields the following expression for $p_{\vartheta}(\theta)$

$$
\begin{aligned}
p_{\vartheta}(\theta)= & \exp \left[A^{2}\left(\frac{g^{2}\left(\theta_{0}, \theta\right)}{4 h(\theta)}-h\left(\theta_{0}\right)\right)\right] \\
& \frac{(2 h(\theta))^{-1}}{2 \pi \sigma_{1} \sigma_{2}}\left[\frac{\pi}{2} \exp \left(-\frac{A^{2} g^{2}\left(\theta_{0}, \theta\right)}{4 h(\theta)}\right)\right. \\
& \left.-\frac{A g\left(\theta_{0}, \theta\right) \sqrt{\pi}}{2 \sqrt{h(\theta)}}\left(1-\Phi\left(\frac{A g\left(\theta_{0}, \theta\right)}{2 \sqrt{h(\theta)}}\right)\right)\right]
\end{aligned}
$$

where $h(\theta)$ and $g\left(\theta_{0}, \theta\right)$ are given by (10) and (11), respectively, and $\Phi(x)$ is the error function defined by $\Phi(x)=$ $\frac{2}{\sqrt{\pi}} \int_{0}^{x} e^{-t^{2}} d t$. Again, it can be verified that (13) reduces to the PDF of the phase process of Rayleigh [10], Rice [12] and Hoyt [5] channels. The influence of the phase shift $\theta_{0}$ on the PDF $p_{\vartheta}(\theta)$ is shown in Fig. 2.

\section{B. Second order statistics}

The second order statistics, given by the LCR and the $\mathrm{ADF}$, describe the correlation properties of the fading channel. They provide useful information for the optimization of the interleaver size [13], the analysis of handoff algorithms [14], [15], the estimation of the statistics of burst errors [16], as well as the Markov modeling of fading channels [17].

The LCR, $N_{R}(r)$, is obtained by applying the general result given by $[10]$

$$
N_{R}(r)=\int_{0}^{\infty} \dot{z} p_{R \dot{R}}(r, \dot{z}) d \dot{z}
$$

where $p_{R \dot{R}}(r, \dot{z})$ is the joint PDF of $R(t)$ and its time derivative $R(t)$ at the same time and at the level $z=r$. This quantity is obtained from (7) as

$$
\begin{aligned}
& p_{R \dot{R}}(z, \dot{z})=\frac{z}{(2 \pi)^{3 / 2}} \int_{0}^{2 \pi} \frac{1}{\sqrt{\beta_{1} \sin ^{2} \theta+\beta_{2} \cos ^{2} \theta}} \\
& \cdot \exp \left[-\frac{z^{2} \cos ^{2} \theta+A^{2} \cos ^{2} \theta_{0}-2 A z \cos \theta_{0} \cos \theta}{2 \sigma_{1}^{2}}\right. \\
& \left.-\frac{z^{2} \sin ^{2} \theta+A^{2} \sin ^{2} \theta_{0}-2 A z \sin \theta_{0} \sin \theta}{2 \sigma_{1}^{2}}\right] \\
& \cdot \dot{z} \exp \left[-\frac{\dot{z}^{2}}{2\left(\beta_{1} \cos ^{2} \theta+\beta_{2} \sin ^{2} \theta\right)}\right] d \theta .
\end{aligned}
$$

It can be seen that $p_{R \dot{R}}(z, \dot{z}) \neq p_{R}(z) \cdot p_{\dot{R}}(\dot{z})$, and therefore $R(t)$ and $\dot{R}(t)$ are statistically dependent. This property was also noted for the Hoyt process [6] and results from the fact that $\sigma_{1} \neq \sigma_{2}$. Now, substituting (15) in (14) and performing the integration yields

$$
\begin{aligned}
N_{R}(r)= & \frac{r(2 \pi)^{-3 / 2}}{\sigma_{1} \sigma_{2}} \int_{0}^{2 \pi} \sqrt{\beta_{1} \cos ^{2} \theta+\beta_{2} \sin ^{2} \theta} \\
& \cdot \exp \left[-\frac{\left(r \cos \theta-A \cos \theta_{0}\right)^{2}}{2 \sigma_{1}^{2}}\right. \\
& \left.-\frac{\left(r \sin \theta-A \sin \theta_{0}\right)^{2}}{2 \sigma_{2}^{2}}\right] d \theta .
\end{aligned}
$$

The ADF, $T_{R-}(r)$, can be computed according to [10]

$$
T_{R-}(r)=\frac{\int_{0}^{r} p_{R}(z) d z}{N_{R}(r)}
$$

In the next section, the fitting of the statistics of the model to available measurement data is studied.

\section{APPLiCATION OF THE GENERALIZED RICE MODEL}

The statistical parameters characterizing the generalized Rice fading model are optimized in such a sense that the first and second order statistics approximate corresponding measured statistics of mobile satellite channels for a light shadowing environment [18]. We consider, in this paper, the Clarke's isotropic scattering model [10]. In this case, $\beta_{i}=$ $2\left(\pi \sigma_{i} f_{\text {max }_{i}}\right)^{2}(i=1,2)$, where $f_{\text {max }_{i}}$ is the maximum Doppler frequency of the Gaussian process $\mu_{i}(t)(i=1,2)$. In other words, we assume different maximum Doppler frequencies $f_{\max _{1}}$ and $f_{\max _{2}}$ for the Gaussian processes $\mu_{1}(t)$ and $\mu_{2}(t)$, respectively. Although, the underlying assumption lacks of a clear physical basis, it allows to increase the flexibility of the model and enables therefore a better fitting to measurement data. The complementary cumulative distribution function (CDF) given by $p_{R+}(r)=\int_{r}^{\infty} p_{R}(z) d z$ and the LCR (16) are compared to corresponding measurement data in Figs. 3 and 4. The optimized parameter values are also shown in these figures. As can be seen from Fig. 3, the complementary CDF is close to the measurement data. The analytical normalized LCR $N_{R}(r) / f_{\max }$, where $f_{\max }=f_{\max }=\sqrt{\beta_{1}} /\left(\sqrt{2} \pi \sigma_{1}\right)$, is compared with measurement data in Fig. 4. The coincidence between the theoretical and experimental data is fairly sufficient and it is comparable to the results obtained in [2], [19] where the same measurement data have been used for the verification of fading channel models. This allows to conclude, in addition to the results of [7], [8], that the generalized Rice model is useful for the description of realistic mobile fading channels. The generalized Rice factor, $K$, can be obtained for the present realistic channel by evaluating (3) using the optimized parameters listed in Fig. 3 . The obtained result is $K=5.55 \mathrm{~dB}$.

For completeness, we should add that the generalized Rice fading model is convenient for, for example, simulation studies. In fact, the well known filter method and the concept of deterministic channel modeling [2] can be directly applied to simulate the envelope fading $R(t)$ and the phase process $\vartheta(t)$.

\section{CONCLUSION}

In this paper, first and second order statistics of the generalized Rice fading channel model have been derived. The derived quantities are fitted to published measurement data on mobile satellite channels for the case of an environment with light shadowing. Close agreement between the theoretical expressions and the measurement data is obtained. This allows to conclude that the generalized Rice model can be applied to the description of realistic mobile satellite channels. Among the advantages of the underlying model is the simplicity of its simulation by using the classical filter method or the concept of deterministic channel modeling. 


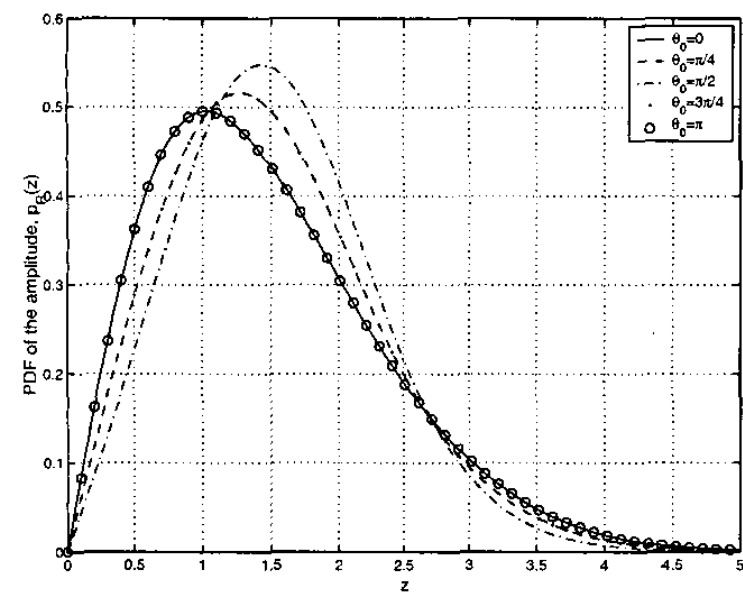

Fig. i. The PDF $p_{R}(z)$ as a function of $\theta_{0}\left(A=1.0, \sigma_{1}^{2}=1.5\right.$ and $\sigma_{2}^{2}=0.5$ ).

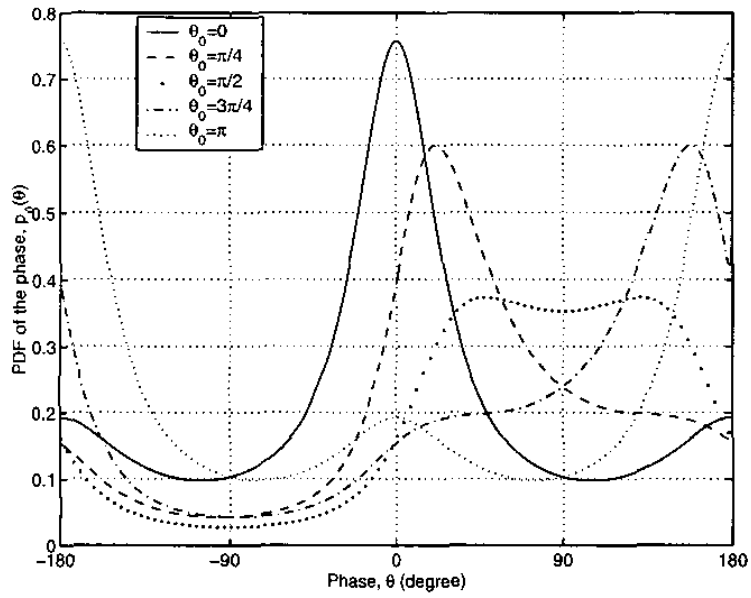

Fig. 2. The PDF $p_{\vartheta}(\theta)$ as a function of $\theta_{0}\left(A=1.0, \sigma_{1}^{2}=1.5\right.$ and $\sigma_{2}^{2}=0.5$ )

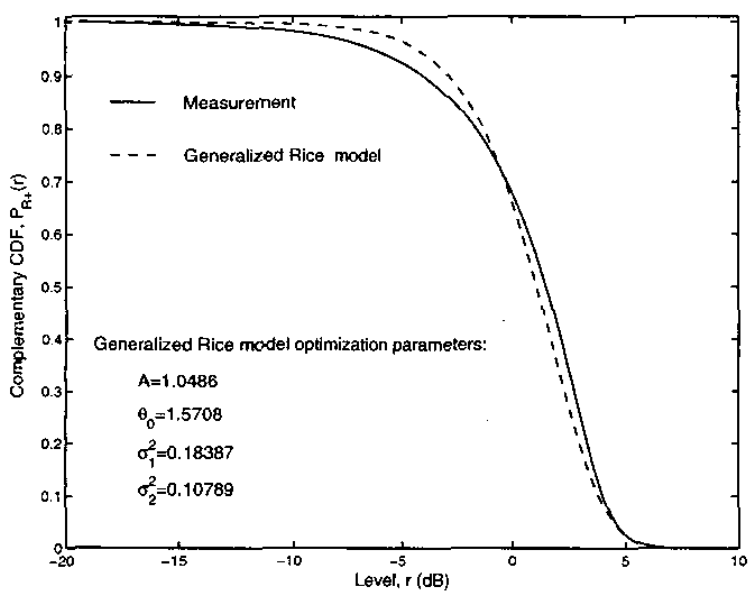

Fig. 3. Complementary CDF $P_{R+}(r)$ for light shadowing.

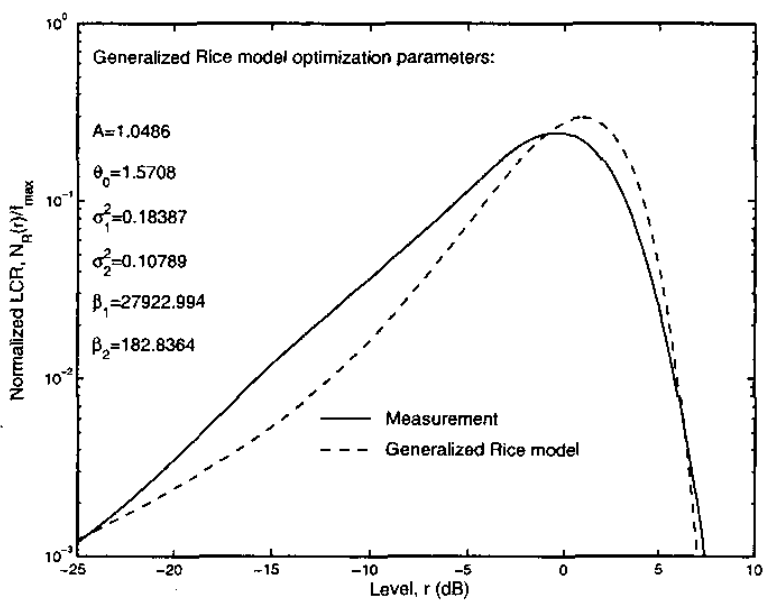

Fig. 4. Normalized LCR $N_{R}(r) / f_{\max }$ for light shadowing.

\section{REFERENCES}

[1] J. D. Parsons, The Mobile Radio Propagation Channel, London: Pentech Press, 1992.

[2] M. Pätzold, Mobile Fading Channels, New York: Wiley, 2002

[3] H. Hashemi, "The indoor radio propagation channel." Proc. IEEE, vol. 81, no. 7. pp. 943-968, July 1993 .

[4] A. Krantzik and D. Wolf, "Distribution of the fading time intervals of modified Suzuki processes," Signal processing V: theory and applications, pp. 361-364, 1990.

[5] R. S. Hoyt, "Probability functions for the modulus and angle of the normal complex variate," Bell Syst. Tech. J., vol. 26. pp. 318-359. April 1947.

[6] N. Youssef, C. X. Wang, and M. Pätzold, "A study on the second order statistics of Nakagami-Hoyt mobile fading channels," IEEE Trans. Veh. Technol., revised version submitted.

[7] P. Beckmann and A. Spizzichino, The scattring of electromagnetic waves from rough surfaces, Norwood, MA: Artech, 1987.

[8] Y. Xie and Y. Fang, "A general statistical channel model for mobile satellite systems," IEEE Trans. Veh. Technol., vol. 49, no. 3. pp. 744752 , May 2000

[9] M. K. Simon and M. S. Alouini, "A unified approach to performance analyis of digital communications over generalized fading channels," in Tutorials notes in VTC, VTC02 Fall, Vancouver, September 2002

[10] W. C. Jakes, Micnowave Mobile Communications. Piscataway, NJ: IEEE Press, 2nd edition, 1993.

[11] I. S. Gradshteyn and I. M. Ryzhik, Table of Integrals, Series, and Products, Academic Press, fifth edition, 1994.

[12] W. B. Davenport and W. L. Root, $A$ introduction to the theory of random signals and noise, New York: McGraw-Hill. 1958.

[13] K. Ip Chan and J. C-I Chuang, "Required interleaving depth in Rayleigh fading channels," in Global Telecommunications Conference, 1996, pp. $1417-1421$

[14] R. Vijayan and J. M. Hotzmann, "Foundations for level crossing analysis of handoff algorithms," in $I C C^{\prime} 93$, May 1993, pp. 935-939.

[15] C. Tepedelenlioglu, A. Abdi, G. B. Giannakis, and M. Kaveh, "Estimation of doppler spread and signal stength in mobile communications with applications to handoff and adaptive transmission," Wirel. Commun. Mob. Comput., pp. 221-242, 2001.

[16] K. Ohtani, K. Daikoku, and H. Omori, "Burst error performance encountered in digital land mobile mobile radio channel," IEEE Trans. Veh. Technol, vol. VT-23, no. 1, pp. 156-160, 1981.

[17] H. S. Wang and N. Moayeri, "Finite state Markov channel - A useful model for radio communication channels," IEEE Trans. Veh. Technol. vol. VT-44, no. 1, pp. 163-171, February 1995.

[18] J. S. Butterworth and E. E. Matt, "The characterization of propagation effects for land mobile satellite services." in in Inter. Conf. Satellite Systems for mobile Commun. Navigationss, June 1983, pp. 51-54.

[19] A. Abdi, W. C. Lau. M. S. Alouini, and M. Kaveh, "A new simple model for land mobile satellite channels: first and second order statistics." Trans. wireless Communu., vol. 2, no. 3, pp. 519-528, May 2003. 